\title{
FOR A CLASS OF AUTONOMOUS DYNAMICAL SYSTEMS
}

\author{
Boro M. Piperevski
}

\begin{abstract}
This paper considers a class of autonomous dynamical systems in many aspects. It shows the connection with EPGLT and EPGD, associated with three type's diffeomorphism. It studied attractor (attractors) for the solution (solutions) at some special autonomous dynamical systems classes. By that, it shows some important properties from topological and metric aspect.
\end{abstract}

\section{INTRODUCTION}

Mathematical formalization of the notion of deterministic process leads to the notion of EPGT (one-parameter group transformations). Here are reviewing EPGD (one-parameter group diffeomorphisms) and their relation to dynamical systems, vector fields, phase flow, phase space, phase curves, autonomous systems, differential equations, etc.

All definitions and theorems in this section are taken from references.

\section{One-parameter group of transformations (EPGT), EPGD, EPGLT}

Definition 1. Let $M$ be a set. A family of transformations of $M$ into itself, is called a group of transformations, if together with any transformation $f$, its inverse transformation $f^{-1}$ belongs to the family, and every two transformations $f$ and $g$, their product fog belong to the family, where $(f \circ g)(x)=f(g(x))$.

Definition 2. Let $G$ be an abstract group and $M$ be a set. We say that a set of group $G$ acts on the set $M$, if to every element $g$ of the group $G$ corresponds to the transformation $T_{g}: M \rightarrow M$, wherein $\forall f, g \in G, \exists T_{f}, T_{g}: M \rightarrow M$, so that

$$
T_{f \circ g}=T_{f} * T_{g}, T_{f^{-1}}=\left(T_{f}\right)^{-1} .
$$

The transformation $T_{g}$ is called the action element $g \in G$ into set $M$. With action $g$ into $m$ is obtained element $T_{g} m=g m$. The set $\{g m \mid g \in G\} \subseteq M$ is called the orbit for the fixed point $m$.

2010 Mathematics Subject Classification. Primary: 37G35, 34C23, Secondary: 34K18.

Key words and phrases. One-parameter group diffeomorphisms (EPGD), phase flow, phase space, dynamical systems, autonomous systems differential equations, Lorenz system 
The action yet determined mapping $T: G \times M \rightarrow M$, i.e. $(g, m) \rightarrow T_{g} m$.

Note: The action of the group $G$ into the set $M$ is the homomorphism of group $G$ in the group transformations of the set $M$.

All processes today are related to time (deterministic process). Therefore, when we talk about the processes, they will be provided with links with time (mathematically speaking with parameter), with the help of a group of real numbers.

Definition 3. Let $G$ be a commutative group and let $R(+)$ is the group of real numbers. The action (or homomorphism) of the $R$ into $G$ is called a one-parameter group $G^{t}=\left\{g^{t} \mid g \in G, t \in R\right\}$. Then $g^{t+s}=g^{t} \circ g^{s}, g^{-t}=\left(g^{t}\right)^{-1}$ is true.

Definition 4. A family $\left\{g^{t}\right\}$ of mappings of a set $M$ into itself, labeled by the set of all real numbers $(t \in R)$, is called a one-parameter group of transformations (EPGT) of $M$, if $g^{t+s}=g^{t} \circ g^{s}$, for all $t, s \in R$ and $g^{0}$ is the identity mapping (which leaves every point fixed). Usually the parameter $t$ is called time, and the Transformation $g^{t}$, is called transformation for time $t$.

If $g^{t}$ is a linear transformation of a set $M$ into itself, then an $\left\{g^{t}\right\}$ is called a oneparameter group of linear transformations (EPGLT).

Definition 5. By a one-parameter group $\left\{g^{t}\right\}$ of diffeomorphism (EPGD) of a manifold $M$ (which can be thought of as a domain in Euclidean space) is meant a mapping

$g: R \times M \rightarrow M, g(t, x)=g^{t} x, t \in R, x \in M$ of the direct product $R \times M$ into $M$ such that

1) $g$ is a differentiable mapping;

2) The mapping $g^{t}: M \rightarrow M$ is a diffeomorphism for every $t \in R$;

3) The family $\left\{g^{t}, t \in R\right\}$ is a one-parameter group of transformations of $M$.

\section{Phase space, phase flow, kinematic and geometric aspect, equivalence of linear flows, Definition of dynamical systems (topological)}

EPGT is mathematically equivalent physical ideas of two-sided deterministic process.

Definition 6. A pair $\left(M,\left\{g^{t}\right\}\right)$ consisting of a set $M$ and one-parameter group $\left\{g^{t}\right\}$ of transformations (EPGT) of $M$ into itself is called a phase flow. The set $M$ is called the phase space of the flow, and its elements are called phase points. In other words phase flow is the set of mappings $g^{t} x: R \times M \rightarrow M$. The orbits of the phase flow are called phase curves or trajectories.

Definition 7. Let $\left(M,\left\{g^{t}\right\}\right)$ be a phase flow, given by a one-parameter group of diffeomorphisms $(E P G D)$ of a manifold $M \subseteq R^{n}$. By the phase velocity $v(\boldsymbol{x})$ of the flow $g^{t}$ at a point $\boldsymbol{x} \in M$ is meant the vector representing the velocity of motion of the phase point, i.e. 


$$
v(x)=\left.\frac{d}{d t}\right|_{t=0}\left(g^{t} x\right) .
$$

The left-hand side of (1.1) is often denoted by $\boldsymbol{x}$ '. Note that the derivative is defined, since the motion is a differentiable mapping of a domain in Euclidean space.

Let $M$ be a domain in Euclidean space with coordinates $x_{1}, x_{2}, \ldots, x_{n}\left(x_{i}: M \rightarrow R\right)$ and suppose that with every point $\boldsymbol{x} \in M$ there is associated the vector $\boldsymbol{v}(\boldsymbol{x})$ emanating from $\boldsymbol{x}$. Then this defines a vector field $\boldsymbol{v}$ on $M$, specified in the $x_{i}$ coordinate system by $n$ differentiable functions $v_{i}: M \rightarrow R$.

Note: In the theory of dynamical systems and topology, dynamical systems are defined by the following definition.

Definition 8. Let $X$ be a topological space and let $F: X \mathrm{x} R \rightarrow X$ is continuous mapping with the following properties:

1. $F(x, 0)=x, \forall x \in X$,

2. $F(x, t+s)=F(F(x, t), s), x \in X, \forall t, s, \in R$

Then the pair $(X, F)$ is called continuous dynamical system (flow), and $X$ is called phase space. The mapping $F_{t}: X \rightarrow X, F_{t}(x)=F(x, t), \forall t \in R$ is called transformation of $X$ at time $t$.

Note: If $F(x, t)=g^{t} x$, then $F(x, t+s)=g^{t+s} x=g^{t}\left(g^{s} x\right)=F\left(g^{t} x, s\right)=F(F(x, t), s)$ is true.

Definition 9. Let $\boldsymbol{x} \in M$ be any phase point, and consider the mapping

$$
\phi: R \rightarrow M, \quad \phi(t)=g^{t} x
$$

of the real line into phase space. Then the mapping (1.2) is called the motion of the point $x$ under the action of the flow $\left(M,\left\{g^{t}\right\}\right)$.

Definition 10. The image of $R$ under the mapping (1.2) is called a phase curve of the flow $\left(M,\left\{g^{t}\right\}\right)$. The graph of the motion (1.2) is called an integral curve of the flow $\left(M,\left\{g^{t}\right\}\right)$.

Theorem 1. Let $M$ be a smooth manifold, and let $v: M \rightarrow T M$ be a vector field. Moreover, let the vector $\boldsymbol{v}(\boldsymbol{x})$ be different from the zero vector of $T M_{x}$ only in a compact subset $K$ of the manifold $M$. Then there exists an EPGD $g^{t}: M \rightarrow M$ for which $v$ is the phase velocity field:

$$
\frac{d}{d t} g^{t} x=v\left(g^{t} x\right)
$$

Corollary 1. Every vector field $\boldsymbol{v}$ on a compact manifold $M$ is the phase velocity field of a EPGD.

Each of these classifications is based on some equivalence relation. There exist at least three reasonable equivalence relations for linear systems, corresponding to algebraic, differentiable, and topological mappings. 
Definition 11. Two phase flow $\left\{g^{t}\right\},\left\{f^{t}\right\}: R^{n} \rightarrow R^{n}$ are said to be equivalent if there exists a one-to-one mapping $h: R^{n} \rightarrow R^{n}$ carrying the flow $\left\{f^{t}\right\}$ into the flow $\left\{g^{t}\right\}$ such that $h \circ f^{t}=g^{t} \circ h$ for every $t \in R$. Under these conditions, the flows are said to be:

1) linearly equivalent if the mapping $h: R^{n} \rightarrow R^{n}$ in question is a linear automorphism;

2) Differentiably equivalent if the mapping $h: R^{n} \rightarrow R^{n}$ is a diffeomorphism;

3) Topologically equivalent if the mapping $h: R^{n} \rightarrow R^{n}$ is a homeomorphism, i.e., if $h$ is one-to-one and continuous in both directions.

Remark 1. Linear equivalence implied differentiable equivalence, while differentiable equivalence implies topological equivalence.

Remark 2. Note that the mapping $h$ carries phase curves of the flow $\left\{f^{t}\right\}$ into phase curves of the flow $\left\{g^{t}\right\}$.

\section{Relationship between EPGD and systems differential equations, the notion of autonomy, EPGLT and linear systems differential equations}

One process, if is defined by the phase flow, changes from one state to another state continuous. Then continuous changes, if given by a vector field $\boldsymbol{v}(\boldsymbol{x})$, can be characterized by derivatives that we can come to the system differential equations $\boldsymbol{x}^{\prime}=\boldsymbol{v}(\boldsymbol{x})$. Because is a concept for a deterministic process (past- present - future), here we are dealing with a stationary process i.e. autonomous system differential equations.

Theorem 2. Let $\left(\left\{g^{t}\right\}, M \subseteq R^{n}\right)$ be the phase flow. Let $\boldsymbol{x}_{0} \in M$ be fixed point and let him consider the mapping $\varphi: R \rightarrow M$, defined with $\varphi(t)=g^{t} \boldsymbol{x}_{0}$. The mapping $\varphi$ occurs as the solution of the system differential equations $\boldsymbol{x}^{\prime}=\boldsymbol{v}(\boldsymbol{x})$ (autonomous system) with initial condition $\varphi(0)=\boldsymbol{x}_{0}$, where

$$
v(x)=\left.\frac{d}{d t}\right|_{t=0}\left(g^{t} x\right) .
$$

Definition 12. Phase flow of the system differential equations $\boldsymbol{x}^{\prime}=\boldsymbol{v}(\boldsymbol{x}), \boldsymbol{x} \in M \subseteq R^{n}$, called EPGD (dynamical system) which occurs as the phase vector field of speeds.

Theorem 3. Let $\boldsymbol{x}^{\prime}=\boldsymbol{v}(\boldsymbol{x}), \boldsymbol{x} \in M \subseteq R^{n}$ is the autonomous system differential equations. Let $M$ be a smooth manifold, and let $v: M \rightarrow T M$ be a vector field. Moreover, let the vector $\boldsymbol{v}(\boldsymbol{x})$ be different from the zero vector of $T M_{x}$ only in a compact subset $K$ of the manifold $M$. Then there exists an EPGD, $g^{t}: M \rightarrow M$ for which $v$ is the phase velocity field:

$$
\frac{d}{d t} g^{t} x=v\left(g^{t} x\right)
$$

In particular, under the conditions of the theorem 1 or those of Corollary 1, we have: 
Corollary 2. Every solution of the system differential equations $\boldsymbol{x}=\boldsymbol{v}(\boldsymbol{x}), \boldsymbol{x} \in M, M$ compact manifold, can be extended indefinitely forward and backward, with the value of the solution $g^{t} \boldsymbol{x}$ at time $t$ depending smoothly on $t$ and the initial condition $\boldsymbol{x}$.

Theorem 4. The family of linear operator $\mathrm{e}^{t A}: R^{n} \rightarrow R^{n}, t \in R$ is an EPGLT of $R^{n}$.

Theorem 5. Let $g^{t}: R^{n} \rightarrow R^{n}$ be a EPGLT. Then there exists a linear operator A: $R^{n} \rightarrow R^{n}$ such that $g^{t}=e^{t A}$.

Theorem 6. The solution of linear autonomous system of differential equations $\boldsymbol{x}^{\prime}=A \boldsymbol{x}$, $\boldsymbol{x} \in R^{n}$, satisfying the initial condition $\varphi(0)=\boldsymbol{x}_{0}$, is given by formula $\varphi(t)=\mathrm{e}^{A t} \boldsymbol{x}_{0}, t \in R$. Thus, adequate EPGLT of the linear autonomous system of differential equations $\boldsymbol{x}^{\prime}=$ Ax is given by $g^{t}=\mathrm{e}^{A t}$ and way around.

Topological linear dynamical system is actually EPGLT and equivalence relation defined in Definition 11 can be transferred to the appropriate linear autonomous systems differential equations.

Theorem 7. Let $A, B: R^{n} \rightarrow R^{n}$ be linear operators all whose eigenvalues are simple. Then the systems $\boldsymbol{x}^{\prime}=A \boldsymbol{x}, \boldsymbol{x} \in R^{n}, \boldsymbol{y}^{\prime}=B \boldsymbol{y}, \boldsymbol{y} \in R^{n}$ are linearly and differentiably equivalent, if and only if the eigenvalues of the operators $A$ and $B$ coincide.

Theorem 8. A necessary and sufficient condition for topological equivalence of two linear systems, all of whose eigenvalues have nonzero real parts, is that the number of eigenvalues with negative (and hence positive) real parts be the same in both systems.

Remark 3. A similar result holds locally (in a neighborhood of a fixed point) for nonlinear autonomous systems whose linear parts have no purely imaginary eigenvalues. In particular, in a neighborhood of a fixed point such a system is topologically equivalent to its linear part.

\section{Correlation of the theory of differential equations with dynamical systems about topological aspect, Attracting sets, attractors}

In the theory of differential equations is known fundamental theorem of existence and uniqueness of the solution of system differential equations. Based on this theorem, shall be proved claims about a separate class of autonomous systems differential equations.

Theorem 9. Let be a given autonomous system differential equations:

$$
\frac{d x_{i}}{d t}=f_{i}\left(x_{1}, x_{2}, \ldots, x_{n}\right), \quad i=1, n,
$$


And let is $\boldsymbol{x}=\varphi\left(t ; \boldsymbol{x}_{0}\right)$, a solution of this system, with initial condition $\varphi(0)=\boldsymbol{x}_{0}$. Then solution $\boldsymbol{x}=\varphi\left(t ; \boldsymbol{x}_{0}\right)$, satisfies the following simple properties:

1) A solution is continuous in terms of the set of all variables,

2) $\varphi\left(0 ; \boldsymbol{x}_{0}\right) \equiv \boldsymbol{x}_{0}$,

3) $\varphi\left(t_{1}+t_{2} ; \boldsymbol{x}_{0}\right) \equiv \varphi\left(t_{2} ; \varphi\left(t_{1} ; \boldsymbol{x}_{0}\right)\right)$.

Note. $\varphi\left(t ; \boldsymbol{x}_{0}\right)=g^{t} \boldsymbol{x}_{0}$ is true.

Property 3 ) is proved by help the fundamental theorem of existence and uniqueness of the solution of the autonomous system differential equations.

Same property in the theory of topology and dynamical systems is used as a condition in definition of dynamical systems. On the other hand, this condition followed by the corresponding condition of the group EPGT.

Definition 13. Let $\varphi(t)$ is a solution of the system differential equations $\boldsymbol{x}^{\prime}=\boldsymbol{v}(\boldsymbol{x})$, $\boldsymbol{x} \in M \subseteq R^{n}$, satisfying the initial condition $\varphi\left(t_{0}\right)=\boldsymbol{x}_{0}$, with defined values for all $t>t_{0}$, and remains for they values $t$ into the closed and bounded set $F \subseteq M$. Point $p$ of the space $M$ is called the $\omega$-the limit point for solution $\varphi(t)$, if there is such a unlimited growing sequence of values (greater than $\left.t_{0}\right) t_{1}, t_{2}, ., t_{k} . ., \lim _{k \rightarrow \infty} t_{k}=\infty$ so that $\lim _{k \rightarrow \infty} \phi\left(t_{k}\right)=p$. The family (totality) $\Omega$ of all limit points of the solutions $\varphi(t)$ is called $\omega$-limit set.

It is shows that the set $\Omega$ is non empty, is closed, is bounded and consists of complete trajectories purposes. The latter means that if the point $\xi$ belongs to $\Omega$, then trajectory of the solution $\varphi(t ; \xi)$ with initial value $\varphi(0)=\xi$, defined for all values of $t$, and whole trajectory of the solution $\varphi(t ; \xi)$, belongs to the set $\Omega$. Obviously that $\omega$-limit set trajectories $\varphi(t ; \xi)$ completely are contained in $\Omega$.

The term the limit-points or limit-set can be replaced with the attracting points or attracting set. A special type such Sets are attractors which, also, exist in different species (global, whimsical, etc.). Sure they are taught the theory of topology, functional analysis and other areas in which systems are studied depending on the change of parameters and their stability.

One construction of a strange attractor - is a product of the Cantor set of manifold. Strange attractor is not a point, orbit, or of type Eight.

Usually today under attractor means minimal, invariant and compact set. Usually strange attractor means set has zero measure 0 and has fractal structure.

In the theory of differential equations these autonomous systems, are sub systems of general Systems differential equations. They are studied by other aspects such as their solving, stability of the solutions (stability theory, Lyapunov functions), the dependence of the behaviour of the system by changing the parameters (the behaviour of solution of a system differential equations on an infinite time interval, chaos, attractors, etc.), approximately solving with qualitative analysis etc. 


\section{MAIN RESULT, LORENZ MODEL}

Theorem 10. Let be given the Lorenz system

$$
\begin{aligned}
& \dot{x}=\sigma(y-x) \\
& \dot{y}=x(r-z)-y . \\
& \dot{z}=x y-b z
\end{aligned}
$$

Let $(x(t), y(t), z(t))$ be the solution, with the initial conditions $x(0)=a_{0}, y(0)=b_{0}, z(0)=c_{0}$, is developed in a Maclaurin's series

$$
x(t)=\sum_{n=0}^{\infty} \frac{a_{n}}{n !} t^{n}, y(t)=\sum_{n=0}^{\infty} \frac{b_{n}}{n !} t^{n}, z(t)=\sum_{n=0}^{\infty} \frac{c_{n}}{n !} t^{n} .
$$

With direct replacement in system and the equalization of coefficients before corresponding degree are getting the system Difference equations

$$
\begin{aligned}
& a_{n}=\sigma\left(b_{n-1}-a_{n-1}\right) \\
& b_{n}=r a_{n-1}-b_{n-1}-\sum_{i=0}^{n-1}\left(\begin{array}{c}
n-1 \\
i
\end{array}\right) a_{i} c_{n-i-1}, \\
& c_{n}=-b c_{n-1}+\sum_{i=0}^{n-1}\left(\begin{array}{c}
n-1 \\
i
\end{array}\right) a_{i} b_{n-i-1}
\end{aligned}
$$

Suppose that numerous series $\sum_{n=0}^{\infty} a_{n}, \sum_{n=0}^{\infty} b_{n}, \sum_{n=0}^{\infty} c_{n}$ are convergent with sums $A, B, C$, respectively. Then the sums can be obtained by formulas

$$
A=\frac{\sigma B+a_{0}}{1+\sigma}, C=r+\frac{(1+\sigma)\left(b_{0}-2 B\right)}{\sigma B+a_{0}}, \sigma B+a_{0} \neq 0,
$$

And $B$ is a solution, real number, to the equation from the third degree

$$
\begin{aligned}
& \sigma^{2} B^{3}+2 \sigma a_{0} B^{2}+\left[r \sigma(1+\sigma)(1+b)-2(1+\sigma)^{2}(1+b)-a_{0}^{2}-c_{0}(1+\sigma) \sigma\right] B+ \\
& +(1+\sigma)(1+b) r a_{0}+(1+\sigma)^{2} b_{0}(1+b)-c_{0}(1+\sigma) a_{0}=0
\end{aligned}
$$

Proof. From system (2.3) by summing up the left and right sides for the $n=1$ to $\infty$ and using the assumption for a convergence of numerous series, is gets the system

$$
A=\sigma(B-A)+a_{0}, B=r A-B-A C+b_{0}, C=-b C+A B+c_{0},
$$

from that is obtained the formula (2.4).

Now, we will apply Remark 3 and Theorem 8, in Lorenz system (2.1) and Rössler system:

$$
\begin{aligned}
& \dot{x}=-(y+z) \\
& \dot{y}=x+\alpha y \\
& \dot{z}=b+x z-c z .
\end{aligned}
$$

First the Rössler system with shift

$$
x=x_{1}+\alpha D, y=y_{1}-D, z=z_{1}+D
$$

It is modified in System 


$$
\begin{aligned}
& \dot{x}=-(y+z) \\
& \dot{y}=x+\alpha y \\
& \dot{z}=x z+\alpha D z+D x-c z,
\end{aligned}
$$

Where $D$ is one of the numbers $\frac{c \pm \sqrt{c^{2}-4 \alpha b}}{2 \alpha}$, and it consider in the neighbourhood of the fixed point (equilibrium position) $O(0,0,0)$. With linearization, own values in the Lorenz system are the roots of the characteristic equation

$$
(\lambda+b)\left[\lambda^{2}+(\sigma+1) \lambda+\sigma-\sigma r\right]=0,
$$

And for the modified Rössler system are the roots of the characteristic equation

$$
\lambda^{3}-A \lambda^{2}-[\alpha(\alpha-A)-2] \lambda-A=0, A=\alpha-c+\alpha D .
$$

Because values in the Lorentz system are real and different for $r>1$, two negative and the third positive, according to observation, the systems are locally topologically equivalence if the characteristic equation (2.5) has two negative and one positive real part of the three roots. In this case in addition to the requirement may be using two modified systems.

The modification of Rössler system has more one equilibrium point

$$
O_{1}\left(c-2 \alpha D, \frac{2 \alpha D-c}{\alpha},-\frac{2 \alpha D-c}{\alpha}\right) \text {. }
$$

Theorem 11. Let be given the Lorenz system differential equations. Let's are consider a corresponding linearization of system in the neighbourhood of the equilibrium position $0(0,0,0)$, given by equations

$$
\begin{aligned}
& \dot{x}=\sigma(y-x) \\
& \dot{y}=r x-y \\
& \dot{z}=-b z
\end{aligned}
$$

The matrix of this system is given by the formula

$$
A=\left[\begin{array}{ccc}
-\sigma & \sigma & 0 \\
r & -1 & 0 \\
0 & 0 & -b
\end{array}\right],
$$

And its own values are roots of the equation

$$
(\lambda+b)\left[\lambda^{2}+(\sigma+1) \lambda+\sigma-\sigma r\right]=0 .
$$

Then the system (2.6) there is an EPGLD given by the formula

$$
g^{t}=\frac{1}{\lambda_{3}-\lambda_{2}}\left[\begin{array}{ccc}
A_{1} & A_{2} & A_{3} \\
B_{1} & B_{2} & B_{3} \\
0 & 0 & e^{\lambda_{1} t}
\end{array}\right],
$$

where

$$
A_{1}=\frac{-r \sigma}{\sigma+\lambda_{2}} e^{\lambda_{2} t}+\frac{r \sigma}{\sigma+\lambda_{3}} e^{\lambda_{3} t}, B_{1}=-r e^{\lambda_{2} t}+r e^{\lambda_{3} t}, A_{2}=\frac{\sigma \beta_{2}}{\sigma+\lambda_{2}} e^{\lambda_{2} t}+\frac{\sigma \gamma_{2}}{\sigma+\lambda_{3}} e^{\lambda_{3} t},
$$




$$
\begin{aligned}
& B_{2}=\beta_{2} e^{\lambda_{2} t}+\gamma_{2} e^{\lambda_{3} t}, A_{3}=\frac{\sigma}{\sigma+\lambda_{1}} e^{\lambda_{1} t}+\frac{\sigma \beta_{3}}{\sigma+\lambda_{2}} e^{\lambda_{2} t}+\frac{\sigma \gamma_{3}}{\sigma+\lambda_{3}} e^{\lambda_{3} t}, B_{3}=e^{\lambda_{1} t}+\beta_{3} e^{\lambda_{2} t}+\gamma_{3} e^{\lambda_{3} t} \\
& \beta_{2}=\frac{\lambda_{2}+\sigma-r \sigma}{\lambda_{2}}, \beta_{3}=\frac{\sigma b^{2}+\sigma b \lambda_{3}+b^{2} \lambda_{2}+\sigma b-r b \sigma}{(\sigma-b) \lambda_{2}}, \gamma_{2}=-\frac{\lambda_{3}+\sigma-r \sigma}{\lambda_{3}}, \gamma_{3}=\frac{b\left(\sigma r-b \lambda_{3}-\sigma \lambda_{2}-\sigma b-\sigma\right)}{(\sigma-b) \lambda_{3}},
\end{aligned}
$$

and $\lambda_{1}=-b, \lambda_{2}, \lambda_{3}$ are roots of the equation (2.7), provided

$$
r \sigma-\sigma+b+b \sigma-b^{2}=0 .
$$

Proof. Until formula (2.8) are coming through eigenvectors of matrix $A$ corresponding to the eigenvalues $\lambda_{1}=-b, \lambda_{2}, \lambda_{3}$ given by the formulas

$$
X_{1}=\left[\begin{array}{c}
\frac{\sigma}{\sigma+\lambda_{1}} \\
1 \\
1
\end{array}\right], X_{2}=\left[\begin{array}{c}
\frac{\sigma}{\sigma+\lambda_{2}} \\
1 \\
0
\end{array}\right], X_{3}=\left[\begin{array}{c}
\frac{\sigma}{\sigma+\lambda_{3}} \\
1 \\
0
\end{array}\right],
$$

and auxiliary matrix

$$
g_{0}^{t}=\left[\begin{array}{ccc}
\frac{\sigma}{\sigma+\lambda_{1}} e^{\lambda_{1} t} & \frac{\sigma}{\sigma+\lambda_{2}} e^{\lambda_{2} t} & \frac{\sigma}{\sigma+\lambda_{3}} e^{\lambda_{3} t} \\
e^{\lambda_{1} t} & e^{\lambda_{2} t} & e^{\lambda_{3} t} \\
e^{\lambda_{1} t} & 0 & 0
\end{array}\right] .
$$

are shows that the corresponding properties

$$
g^{0}=E, g^{t+s}=g^{t} g^{s}, \frac{d}{d t} g^{t} x=v\left(g^{t} x\right),
$$

are valid.

\section{Conclusion}

Usually in the theory of differential equations, autonomous systems are called dynamical Systems differential equations only for the appropriate condition in group EPGD (Definition 4), thus explicitly not is stated that are autonomous. With Definition 12 clearly defines the essential difference between dynamical systems and autonomous systems differential equations. Properties 1-3 of Theorem 9 are as essential.

Often under dynamical systems (flow otherwise) in Definition 8 (conditions 1,2 ) means the family transformations $\varphi\left(t ; x_{0}\right)$ any set into itself (where can be defined continuity transformations), if the properties 1-3 of Theorem 9 are met, even when are not given differential equations.

Discussed the similarities and differences

The Definitions 5, 6, 8, 9, 12, together with Theorems 2, 3 and 9 gives the similarity and difference between the terms dynamical systems and autonomous systems differential equations. Relationships between them are given with the Theorems 1,2 and 3 .

Thus with each EPGD is connected system differential equations (given by the vector field phase speeds) whose solution appears as a moving phase points under the influence of the phase flow. If phase flow describes any process in random initial 
conditions then the system differential equations given by its vector field of the phase speeds determined by the local law of the evolution process. In the theory of differential equations is required, knowing the law of evolution, to conclude the past and predict the future. The formulation of any law of nature in the form of differential equation is reduced any job for the evolution process (physical, chemical, environmental, biological, etc.) in the geometric task for behaviour of phase curves of a given vector field in the appropriate phase space.

Usually today, quite often are mixed dynamical system in terms (topological) and dynamical system autonomous differential equations. Obviously dynamical system (topological) by definition fits only in autonomous system differential equations. The opposite is not necessarily true as shown with the example $\frac{d x}{d t}=x^{2}$.

\section{References}

[1] J. E. Marsden, M. McCracken: The Hopf Bifurcation and its Applications, Springer-Verlag, New York 1976.

[2] G. Ioss, D. D. Joseph: Stability and Bifurcation Theory, Springer - Verlag, New York, Heidelberg, Berlin1980.

[3] Arnold V. I.: Ordinary Differential Equations, MIT1978.

[4] E.L.Ince: Ordinary Differential Equations, Dover Publications INC. New York 1956.

[5] Л. С. Понтрягин: Обыкновеннье дифференциальньее уравнения, Наука, Москва1970.

[6] И. Г. Петровский: Лекц̧ии по теории обыкновенных дифференциальных уравнений, Наука, Москва, 1970.

[7] Schroeder M.: Fractals, Chaos, Power Laws, W.H.Freeman and Co., New York1991.

[8] R. Barrio, F. Blesa, S.Serrano: Behavior patterns in multiparametric dynamical systems: Lorenz model, International Journal of Bifurcation and Chaos, Vol. 22, No. 6 (2012) 1230019

Faculty of Electrical Engineering and Information Technologies, University "Ss Cyril and Methodius", Skopje, Macedonia, E-mail address: borom@feit.ukim.edu.mk 